\title{
Mortality, survival and incidence rates in the ITALUNG randomised lung cancer screening trial
}

\author{
Eugenio Paci, ${ }^{1}$ Donella Puliti, ${ }^{1}$ Andrea Lopes Pegna ${ }^{2}$ Laura Carrozzi, ${ }^{3}$ \\ Giulia Picozzi, ${ }^{4}$ Fabio Falaschi, ${ }^{5}$ Francesco Pistellii, ${ }^{3}$ Ferruccio Aquilini, ${ }^{3}$ \\ Cristina Ocello, ${ }^{1}$ Marco Zappa, ${ }_{1}^{1}$ Francesca M Carozzi, ${ }^{6}$ Mario Mascalchi, ${ }^{7}$ \\ and the ITALUNG Working Group
}

\begin{abstract}
- Additional material is published online only. To view please visit the journal online (http://dx.doi.org/10.1136/ thoraxinl-2016-209825)
\end{abstract}

For numbered affiliations see end of article.

Correspondence to Dr Eugenio Paci, Clinical Epidemiology Unit, ISPOCancer Research and Prevention Institute, Via delle Oblate, Florence 50144, Italy; paci.eugenio@gmail.com

Received 7 December 2016 Revised 6 February 2017 Accepted 9 March 2017

Published Online First 4 April 2017

\section{Linked}

- http://dx.doi.org/10.1136/ thoraxjnl-2017-210268

CrossMark

To cite: Paci E, Puliti D,

Lopes Pegna A, et al. Thorax 2017:72:825-831.

\section{ABSTRACT}

Background ITALUNG is contributing to the European evaluation of low-dose CT (LDCT) screening for lung cancer (LC).

Methods Eligible subjects aged 55-69 years, smokers or ex-smokers (at least 20 pack-years in the last 10 years), were randomised to receive an annual invitation for LDCT screening for 4 years (active group) or to usual care (control group). All participants were followed up for vital status and cause of death (at the end of 2014) and LC incidence (at the end of 2013). Pathological and clinical information was collected from the Tuscan Cancer Registry data.

Results 1613 subjects were randomly assigned to the active group and 1593 to the control group. At the end of the follow-up period $67 \mathrm{LC}$ cases were diagnosed in the active group and 71 in the control group (rate ratio $(R R)=0.93 ; 95 \% \mathrm{Cl} 0.67$ to 1.30). A greater proportion of stage I LC was observed in the active group (36\% vs $11 \%, p<0.001)$. Non-significant reductions of $17 \%$ $(\mathrm{RR}=0.83 ; 95 \% \mathrm{Cl} 0.67$ to 1.03$)$ for overall mortality and $30 \%$ ( $R R=0.70 ; 95 \% \mathrm{Cl} 0.47$ to 1.03 ) for LC-specific mortality were estimated.

Conclusions Despite the lack of statistical significance, the ITALUNG trial outcomes suggest that LDCT screening could reduce LC and overall mortality. Moreover, the comparison of the number of $L C$ cases diagnosed in the two groups does not show overdiagnosis after an adequate follow-up period. A pooled analysis of all European screening trials is advocated to assess the benefit-to-harm ratio of LDCT screening and its implementation in public health settings. Trial registration number Results, NCT02777996.

\section{BACKGROUND}

Lung cancer (LC) is the leading cause of cancer deaths in men and the third in women in Italy, with an increasing incidence and mortality trend among women. Over 33000 LC deaths occurred in 2012 and 41000 LC diagnoses were estimated in 2015 . $^{1}$ Although slightly improving, the 5-year Cancer Registry-based LC survival rate in Italy is still only $14 \% .^{2}$ Both the LC incidence and mortality rates are decreasing among men as a consequence of the reduction in smoking. However, ageing of the Italian population will result in a substantial stability in the absolute number of LC deaths. ${ }^{3} \mathrm{New}$ tobacco control policies have been implemented in

\section{Key messages}

What is the key question?

- After the positive results of the National Lung Screening Trial in the USA, the efficacy of lung cancer screening with low-dose CT (LDCT) is still debatable in Europe.

What is the bottom line?

- Despite the lack of statistical significance, the outcomes of the ITALUNG trial suggest that LDCT screening could reduce lung cancer and overall mortality and does not show overdiagnosis after an adequate follow-up period.

\section{Why read on?}

- The results of the ITALUNG trial, which is part of the European Randomised Trials Collaboration, make an important contribution to the assessment of the benefit-to-harm ratio of lung cancer screening in Europe.

Italy and the promotion of smoking cessation strategies is considered to be the leading primary prevention strategy to reduce smoking-attributable LC deaths. ${ }^{4}$

Screening for LC with low-dose CT (LDCT) in the National Lung Screening Trial (NLST) reduced LC mortality by $20 \%,{ }^{5}$ and the guidelines for screening for LC were rapidly updated so that LDCT screening is currently recommended for high-risk subjects in the USA. ${ }^{6}$ Conversely, public health guidelines in Europe do not recommend screening for LC since the evidence of its benefits and harms has not been considered sufficient. ${ }^{7}$

The ITALUNG LC screening trial was launched in 2004 in Tuscany, an Italian administrative region, with the aim of contributing to the European evaluation of the efficacy of LDCT screening for reducing LC-specific and overall mortality and an assessment of the benefit-to-harm ratio.

\section{METHODS}

ITALUNG is a randomised controlled trial (RCT) of screening for LC, comparing LDCT to usual 
care, carried out in three Tuscan screening centres (Florence, Pisa and Pistoia). The study design and criteria for a positive test were previously reported as the major indicators of screening performance in the active group. ${ }^{89} \mathrm{~A}$ flow chart of the management of non-calcified solid, part-solid and non-solid nodules detected at baseline or repeat LDCT screening is shown in the online supplementary appendix (section 1).

Subjects recruited from the patient lists of 269 general practitioners were sent a letter with a standardised questionnaire which they were requested to return by post giving consent to be randomised. Subjects were eligible if they were aged 55-69 years with a smoking history of at least 20 pack-years in the last 10 years (former smokers who had quit more than 10 years ago were excluded). As the enrolment was based on the year of birth, a small percentage of subjects were 54 years old at the date of randomisation (but they became 55 during that calendar year); in the same way, subjects aged 69 years at randomisation became older during the calendar year. Other exclusion criteria were a history of previous cancer other than non-melanoma skin cancer and general conditions precluding thoracic surgery. Eligible subjects were centrally randomised by a software procedure into an active group receiving an annual invitation to LDCT screening for 4 years and a control group receiving usual care. Subjects in the active group were given a clinical standardised interview. Control group subjects received a letter communicating their allocation. Both groups received an invitation to a free smoking cessation programme.

\section{Statistical analysis}

The study's primary endpoint was the comparison of LC mortality between the active and control groups using the rate ratio (RR) with 95\% CI. Secondary analyses compared the rate of death from any cause, the rate of death from any cause except LC and the incidence of LC in the two groups.

Power calculation was made assuming pooling of mortality data with other European trials, among which the NELSON trial is the largest. Assuming a $25 \%$ reduction in LC mortality among screened subjects after 10 years of follow-up, the required sample size for a power of $80 \%$ was calculated to be between 15200 and 18700 subjects (depending on the eligibility criteria of the participants). ${ }^{10}$

Subjects who withdrew from the screening process at any time after randomisation were considered as dropouts but, in accordance with the intention-to-treat principle, were included in the active group.

All participants were followed up for vital status and cause of death until 31 December 2014. Follow-up for vital status was performed via the population mortality registries and included a check for residential status. An independent committee reviewed and revised the causes of death in a blinded fashion using a specific algorithm presented in the online supplementary appendix (section 2).

All participants were followed up for the incidence of LC until 31 December 2013 through links to the Tuscan Cancer Registry. In cases of multiple LC diagnoses in the same subject, only the first was considered. All LC patients were classified as either treated with surgery or not, and disease stage was determined on the basis of histology reports (pTNM) or clinical information when histology reports were not available (ie, all non-resected patients and five resected patients with missing histology reports) according to the 7th edition of the TNM classification. ${ }^{11}$ Histological characteristics were coded according to the International Classification of Disease for Oncology, 3rd edition (ICD-O-3). ${ }^{12}$ Morphological characterisation was cytology-based in cases in which neither core biopsy nor surgery were performed.

All active group subjects who were diagnosed with LC were classified as either screen-detected (diagnosis as a result of a screening test or a follow-up test in accordance with the proto$\mathrm{col}$ ), clinically detected while in screening (diagnosis not as a result of screening but the subject had attended at least one screening test) or clinically detected among the unscreened (subjects who did not attend any screening test).

The person-years at risk were counted from the date of randomisation to the date of event (LC diagnosis or death) or to the date of censoring (migration or end of follow-up), whichever came first.

Major complications from invasive diagnostic procedures were monitored and compared between the two groups according to two indicators:

a. mortality within 60 days after the surgical treatment, defined as the proportion of enrolled subjects who died within 60 days after surgical treatment;

b. mortality within 60 days after most invasive diagnostic procedure (surgery, biopsy, bronchoscopy or fine needle aspiration cytology), defined as the proportion of enrolled subjects who died within 60 days after an invasive diagnostic procedure. For patients who did not undergo an invasive procedure, deaths were included if they occurred within 60 days after the diagnosis date.

In the survival analysis we included only study subjects diagnosed before or on 31 December 2013. The median follow-up time from the date of diagnosis was 1.2 years (Q1-Q3: 0.63.4). The 3-year LC survival was estimated using the Kaplan-Meier method and the difference between survival curves was tested using the log-rank test.

\section{RESULTS}

Figure 1 shows subject recruitment and outcomes (LC diagnosed, LC deaths and deaths from other causes) for the active and control groups at the end of the follow-up period. From 2004 to 2006, 71232 invitation letters were sent to subjects aged 55-69 years resident in one of the three districts included in the study. The questionnaire was sent back to the screening centre by 17055 responders, of whom 3206 were eligible to be randomised. Participants were randomly assigned to the active group who received annual LDCT screening for 4 years $(n=1613)$ or to the control group who received usual care $(n=1593)$.

Among subjects allocated to the active group, 1406 underwent the baseline LDCT scan. The 207 dropouts between randomisation and the baseline screening test were mainly due to refusal to undergo baseline LDCT after randomisation (79.2\%). Overall, there was $81 \%$ adherence to the screening protocol across the four LDCT rounds (1302/1613), with both the 1258 subjects who attended all four LDCT rounds and the 44 subjects who attended fewer than four LDCT rounds due to LC diagnosis or death being considered as compliant with the protocol.

The demographic characteristics and smoking habits of the enrolled subjects by study group are presented in table 1 . The distribution of subjects by age, sex and smoking habits (smoking status and pack-years) was well balanced between the two groups.

\section{LC incidence}

The median follow-up time for LC incidence was 8.5 years (Q1-Q3: 7.9-8.9). A total of 67 lung cancers (49.9 per 10000 person-years) were diagnosed in the active group compared 


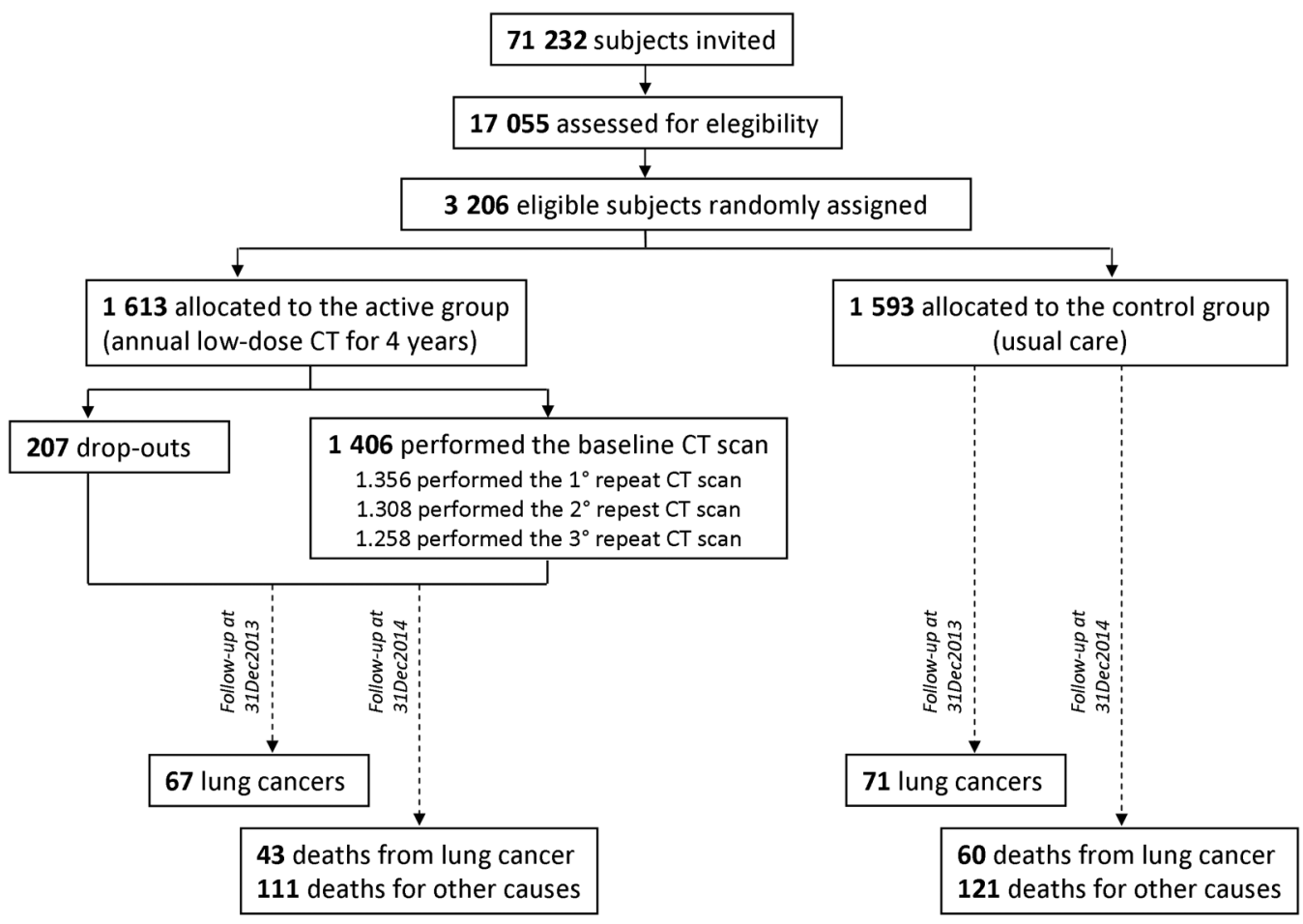

Figure 1 Subject recruitment and outcome of the ITALUNG trial.

Table 1 Characteristics of study participants $(n=3206)$

\begin{tabular}{lll}
\hline & $\begin{array}{l}\text { Active group } \\
(\mathbf{n}=1613)\end{array}$ & $\begin{array}{l}\text { Control group } \\
(\mathbf{n}=1593)\end{array}$ \\
\hline $\begin{array}{l}\text { Centre } \\
\text { Florence }\end{array}$ & $795(49 \%)$ & $783(49 \%)$ \\
$\quad$ Pisa & $395(25 \%)$ & $392(25 \%)$ \\
$\quad$ Pistoia & $423(26 \%)$ & $418(26 \%)$ \\
Age at entry (years) & & \\
$<55$ & $53(3 \%)$ & $64(4 \%)$ \\
$55-59$ & $687(43 \%)$ & $616(39 \%)$ \\
$60-64$ & $497(31 \%)$ & $526(33 \%)$ \\
$65-69$ & $371(23 \%)$ & $382(24 \%)$ \\
$>69$ & $5(0.3 \%)$ & $5(0.3 \%)$ \\
$\quad$ Mean age at entry & 60.9 & 60.7 \\
Sex & & $1039(65 \%)$ \\
$\quad$ Male & $1035(64 \%)$ & $554(35 \%)$ \\
Female & $578(36 \%)$ & $1018(64 \%)$ \\
Smoking status & & $575(36 \%)$ \\
$\quad$ Current & $1059(66 \%)$ & 38 \\
$\quad$ Former & $554(34 \%)$ & \\
Median pack-years of smoking & 40 & \\
\hline
\end{tabular}

with 71 (53.7 per 10000$)$ in the control group $(R R=0.93$; $95 \%$ CI 0.67 to 1.30$)$. Among the LC cases diagnosed in the active group, 38 (57\%) were screen-detected, 25 (37\%) were clinically detected in screened subjects and $4(6 \%)$ were clinically detected in unscreened subjects (ie, those who refused screening immediately after randomisation).

Figure 2 shows the cumulative number of lung cancers diagnosed up until 31 December 2013 per study group. The excess of cases in the active group was $55 \%$ in the first 4 years (the screening period) whereas a $45 \%$ reduction was observed in the
5 years post-screening. The catch-up of the cumulative number of lung cancers took between 6 and 7 years from randomisation.

In table 2 the characteristics of the diagnosed lung cancers are presented per study group. About half of the patients in the active group were treated with surgery compared with a quarter in the control group $(52 \%$ vs $28 \%, \mathrm{p}=0.003)$. Similarly, a greater proportion of stage I cancers was observed in the active group than in the control group ( $36 \%$ vs $11 \%, \mathrm{p}<0.001)$. The distribution by histotype showed a non-significant excess of adenocarcinoma in the active group (43\% vs $30 \%, \mathrm{p}=0.09)$ whereas the proportion of small cell carcinomas was $15 \%$ in both groups. The characteristics of LC diagnosed in the active group by modality of detection (screen-detected, clinically detected among screened and among unscreened) are reported in the online supplementary appendix (section 3).

\section{Overall and LC-specific mortality}

After a median follow-up of 9.3 years (Q1-Q3: 8.8-9.9), the active group showed a non-significant $17 \%$ reduction $(\mathrm{RR}=0.83 ; 95 \%$ CI 0.67 to $1.03 ; \mathrm{p}=0.08)$ in overall mortality and a $30 \%$ reduction $(\mathrm{RR}=0.70 ; 95 \% \mathrm{CI} 0.47$ to 1.03 ; $\mathrm{p}=0.07)$ in LC-specific mortality compared with the control group (table 3 ). There were 154 deaths in the active group and 181 deaths in the control group, corresponding to rates of death from any cause of 105.1 and 127.0 , respectively, per 10000 person years. Among these, 43 deaths from LC (29.3 per 10000 person-years) were observed in the active group compared with 60 (42.1 per 10000$)$ in the control group. The absolute rate difference (per 10000 ) between the active and control groups was 21.9 for overall mortality and 12.8 for LC-specific mortality. Among subjects who died of LC in the active group, 17 (40\%) were screen-detected, 23 (53\%) were clinically detected in screened subjects and $3(7 \%)$ were clinically detected in unscreened subjects. 
Figure 2 Cumulative numbers of lung cancers by year from randomisation per group. The number of lung cancers includes lung cancers that were diagnosed from the date of randomisation through 31 December 2013. The grey area indicates the screening period.

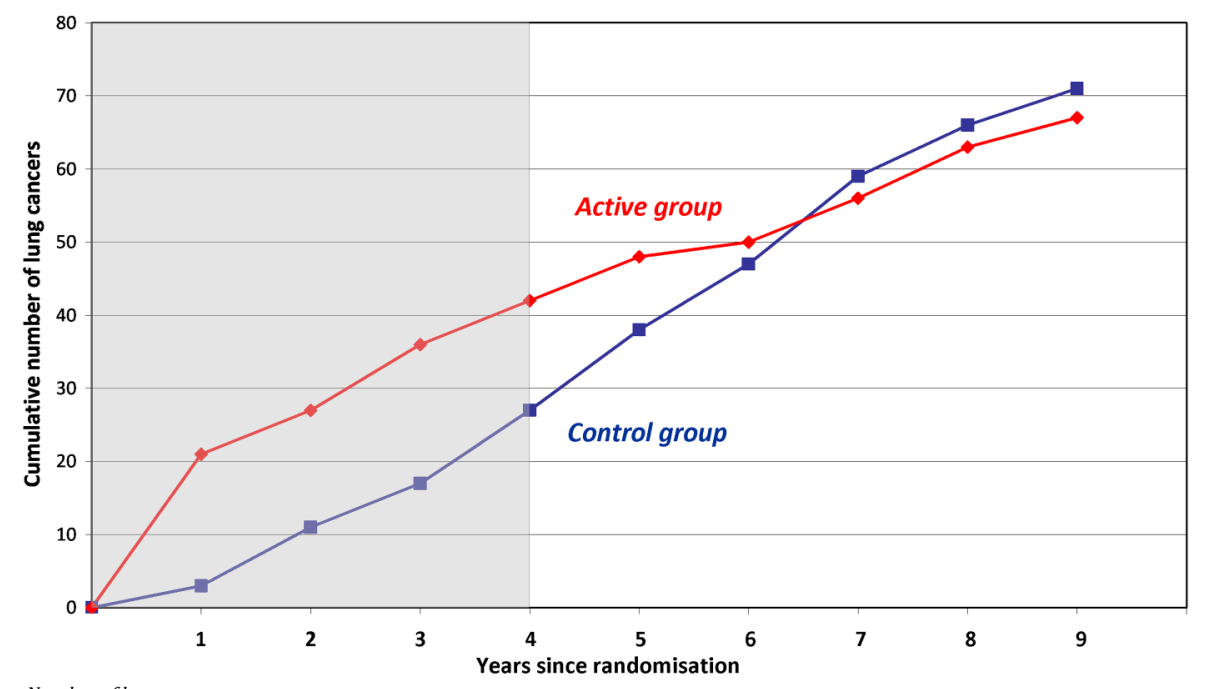

\begin{tabular}{lccccccccc}
\multicolumn{1}{l}{\begin{tabular}{l} 
Number of lung cancer: \\
\hline Control
\end{tabular}} & 3 & 8 & 6 & 10 & 11 & 9 & 12 & 7 & 5 \\
Active & 21 & 6 & 9 & 6 & 6 & 2 & 6 & 7 & 4 \\
\hline
\end{tabular}

The lower overall mortality of the active group fell to a statistically non-significant $11 \%$ when deaths for LC were excluded $(\mathrm{RR}=0.89 ; \mathrm{p}=0.38)$ (table 3$)$. Although the outcomes of the study did not include cause-specific mortality, we observed a statistically significant reduction for cardiovascular mortality $(\mathrm{RR}=0.51 ; \mathrm{p}=0.009)$.

Figure 3 shows the cumulative number of overall deaths and LC deaths by years from randomisation per group. Although overall mortality was quite similar in the two groups during the screening phase $(\mathrm{RR}=0.97 ; \mathrm{p}=0.86)$, a significant $23 \%$ reduction was observed in the active group in the post-screening period $(R R=0.77 ; p=0.045)$. There was a slight non-significant excess of LC-specific mortality in the active group during the screening period $(R R=1.20 ; p=0.62)$ but, in the post-screening

Table 2 Surgery, stage and histological type of lung cancers in the study groups $(n=138)$

\begin{tabular}{|c|c|c|c|}
\hline & $\begin{array}{l}\text { Active group } \\
(n=67)\end{array}$ & $\begin{array}{l}\text { Control group } \\
(n=71)\end{array}$ & p Value \\
\hline \multicolumn{4}{|l|}{ Surgery } \\
\hline Resected & $35(52 \%)$ & $20(28 \%)$ & \\
\hline Not resected & $32(48 \%)$ & $51(72 \%)$ & 0.003 \\
\hline \multicolumn{4}{|l|}{ Stage* } \\
\hline I & $24(36 \%)$ & $8(11 \%)$ & \\
\hline II & $5(7 \%)$ & $5(7 \%)$ & \\
\hline III & $9(13 \%)$ & $8(11 \%)$ & \\
\hline IV & $24(36 \%)$ & $35(49 \%)$ & \\
\hline Unknown & $5(7 \%)$ & $15(21 \%)$ & 0.005 \\
\hline \multicolumn{4}{|l|}{ Histological type } \\
\hline Adenocarcinoma & $29(43 \%)$ & $21(30 \%)$ & \\
\hline Squamous cell carcinoma & $14(21 \%)$ & $17(24 \%)$ & \\
\hline Small cell lung cancers & $10(15 \%)$ & $11(15 \%)$ & \\
\hline Carcinoid & $2(3 \%)$ & $0(0 \%)$ & \\
\hline Non-small cell carcinomat & $3(4 \%)$ & $5(7 \%)$ & \\
\hline Unclassified & $9(13 \%)$ & $17(24 \%)$ & 0.19 \\
\hline
\end{tabular}

period, a significant $46 \%$ reduction $(R R=0.54 ; p=0.01)$ was observed.

\section{Adverse events}

The death rates within 60 days after surgical treatment were 1.2 $(2 / 1613)$ and $1.3(2 / 1593)$ per 1000 in the active and control groups, respectively $(\mathrm{p}=0.99)$. Similarly, the death rates within 60 days after most invasive diagnostic procedure were almost the same in the two groups, $3.7(6 / 1613)$ vs $3.8(6 / 1593)$ per $1000, p=0.98$ (adverse events are listed in section 4 of the online supplementary appendix). Although the two groups had a similar cumulative frequency of adverse events, those in the active group occurred sooner in time due to diagnostic anticipation. Indeed, two out of six deaths-defined as adverse events in the active group-occurred in screen-detected patients.

\section{LC survival}

In total, 99 deaths from any causes (96 from LC and 3 from other causes) were observed among 138 LC diagnoses by 31 December 2013. The 3 -year LC survival was $44 \%$ and $25 \%$ for the active and control groups, respectively $(p=0.07)$. In figure 4 , LC survival curves shown by type of treatment (surgically

Table 3 Mortality rate (per 10000 person-years) and rate ratios by group

\begin{tabular}{lllll}
\hline & $\begin{array}{l}\text { Active } \\
\text { group }\end{array}$ & $\begin{array}{l}\text { Control } \\
\text { group }\end{array}$ & $\begin{array}{l}\text { Rate ratio } \\
(95 \% \mathrm{CI})\end{array}$ & p Value \\
\hline $\begin{array}{l}\text { Person-years* } \\
\text { Overall mortality rate }\end{array}$ & $\begin{array}{l}14658 \\
105.1 \\
(\mathrm{n}=154)\end{array}$ & $\begin{array}{l}14247 \\
127.0 \\
(\mathrm{n}=181)\end{array}$ & $0.83(0.67$ to 1.03) & 0.08 \\
$\begin{array}{l}\text { Lung cancer mortality } \\
\text { rate }\end{array}$ & $\begin{array}{l}29.3 \\
(\mathrm{n}=43)\end{array}$ & $\begin{array}{l}42.1 \\
(\mathrm{n}=60)\end{array}$ & $0.70(0.47$ to 1.03) & 0.07 \\
$\begin{array}{l}\text { Overall mortality rate } \\
\text { except lung cancer }\end{array}$ & $\begin{array}{l}75.7 \\
(\mathrm{n}=111)\end{array}$ & $\begin{array}{l}84.9 \\
(\mathrm{n}=121)\end{array}$ & $0.89(0.69$ to 1.15) & 0.38 \\
$\begin{array}{l}\text { Cardiovascular } \\
\text { disease }\end{array}$ & $\begin{array}{l}15.0 \\
(\mathrm{n}=22)\end{array}$ & $\begin{array}{l}29.5 \\
(\mathrm{n}=42)\end{array}$ & $0.51(0.30$ to 0.85) & 0.009 \\
Other causes & $\begin{array}{l}60.7 \\
(\mathrm{n}=89)\end{array}$ & $\begin{array}{l}55.5 \\
(\mathrm{n}=79)\end{array}$ & $1.10(0.81$ to 1.48) & 0.56 \\
\hline *Follow-up at 31 December 2014. & & & \\
\hline
\end{tabular}



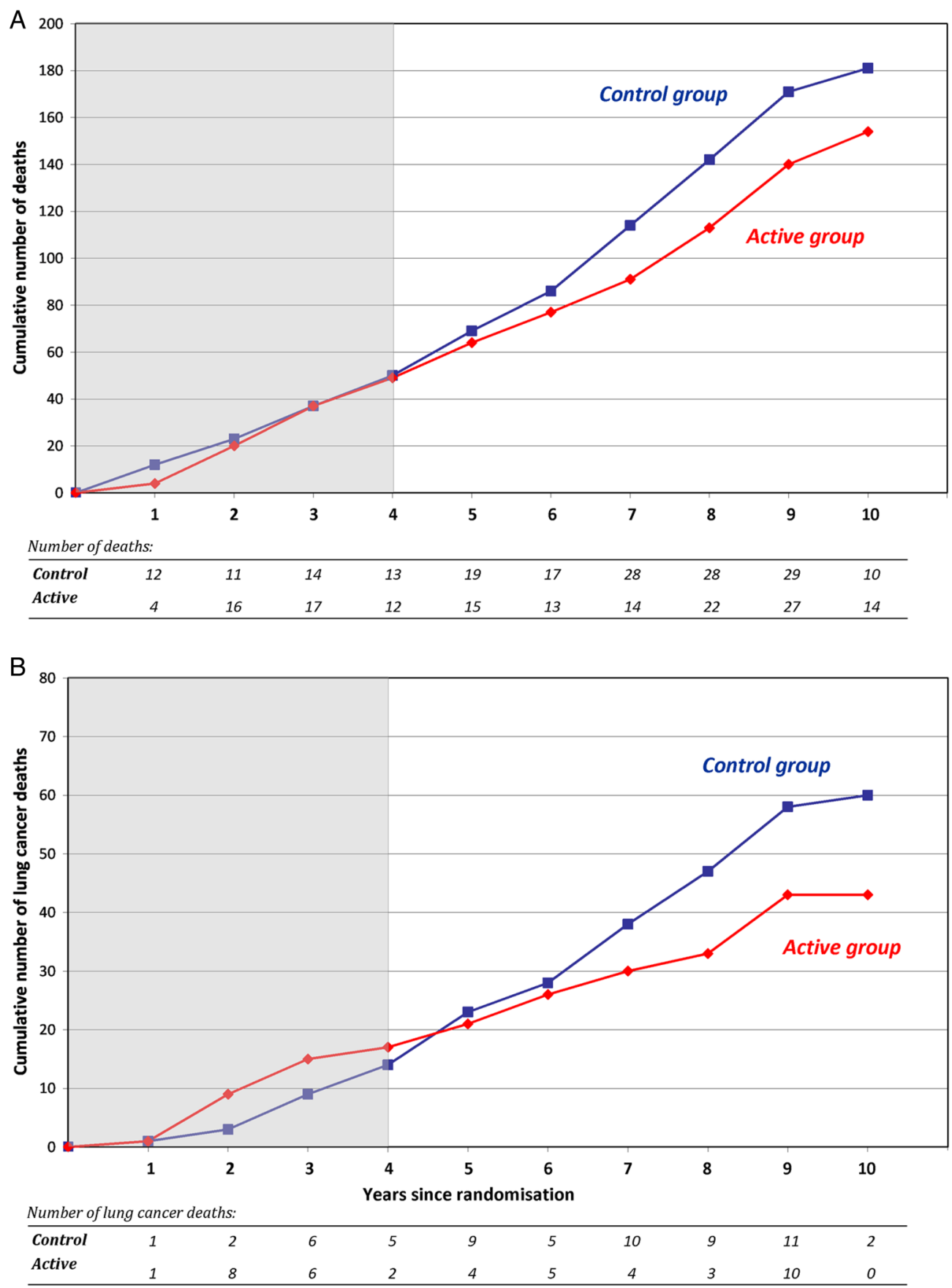

Figure 3 Cumulative numbers of (A) overall deaths and (B) lung cancer deaths by year from randomisation per group. The number of deaths includes deaths that occurred from the date of randomisation through 31 December 2014. The grey area indicates the screening period.

resected/not resected) and study group indicate that there was no difference in 3-year survival between the two groups for patients treated with surgery $(74 \%$ vs $67 \%, \mathrm{p}=0.70)$. Similarly, survival in unresected patients was $7 \%$ and $8 \%$ in the active and control groups, respectively $(\mathrm{p}=0.50)$.

\section{DISCUSSION}

At 9 years of follow-up, non-significant reductions of $30 \%$ in LC-specific mortality $(p=0.07)$ and $17 \%$ in overall mortality $(p=0.08)$ were observed in the group screened with LDCT in comparison with the usual care control group. Despite the lack of statistical significance, we observed a consistent temporal trend. Indeed, the analysis by length of follow-up strengthened these results: no difference in mortality was observed during the screening phase (the 4 years following randomisation) whereas a significant reduction in both LC-specific $(p=0.01)$ and overall mortality $(p=0.045)$ was observed in the post-screening period.

Notably, the decrease in mortality observed in our study was larger than that reported in the $\mathrm{NLST}^{5}$ in which decreases of $20 \%$ and $7 \%$ in LC-specific and overall mortality, respectively, were observed at 6.5 years of median follow-up. Besides the statistical uncertainty due to the different sample sizes of the NLST and ITALUNG studies, several factors must be considered in comparing their mortality data. First, the larger percentage decrease in LC mortality observed in the ITALUNG trial is probably explained by the longer follow-up, as shown from the trend of the mortality curves (the difference between the two groups became evident from the sixth year). Second, in the 


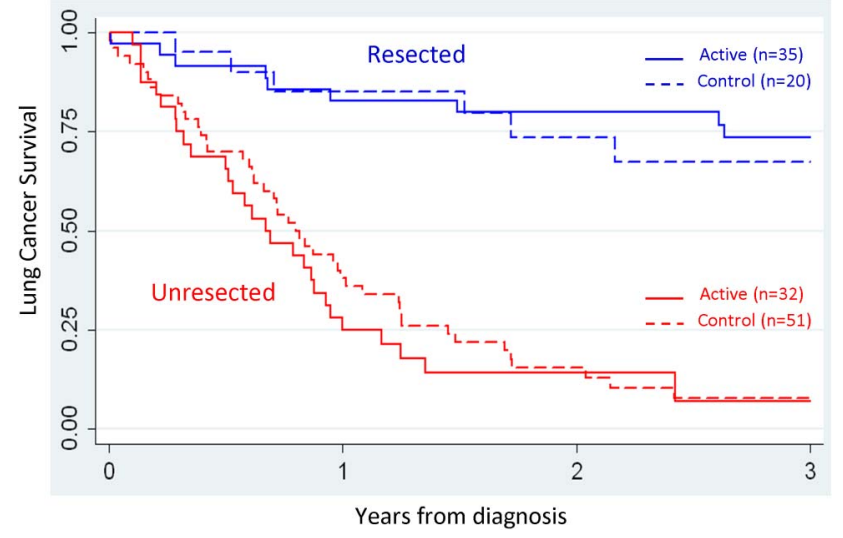

Figure 4 Lung cancer survival curves by surgery and group.

ITALUNG trial, subjects enrolled in the control group were not invited to screening but received usual care, whereas in the NLST the control group underwent chest posterior-anterior (PA) radiography. The proportion of LC cases in the control groups diagnosed at an early stage and treated with surgery was noticeably higher in the NLST than in the ITALUNG trial $(44 \%$ vs $28 \%$ had surgery and $31 \%$ vs $11 \%$ were diagnosed at an early stage in the NLST and ITALUNG trials, respectively) (see NLST online supplementary appendix ${ }^{5}$ ). Third, the eligible subjects enrolled can have different risk profiles for LC, as we have shown by comparing the risk profile and performance of ITALUNG with that of the NLST and the UK Lung Cancer Screening (UKLS) trial. $^{13}{ }^{14}$ The evaluation of risk profiles across studies is a further important motivation for the pooled analysis of LC screening trials. Fourth, it is important to note that only $40 \%$ of the LC deaths in the active group occurred in screen-detected LC cases, a proportion which was influenced by the length of the post-screening phase and which suggests that the effect of LDCT screening could have been even larger.

In the ITALUNG trial the distribution of LC stage differed strongly by group, with $36 \%$ and $11 \%$ of LC cases diagnosed at an early stage in the active and control groups, respectively. Of the LC cases in the active group, 52\% were treated with surgery compared with only $28 \%$ in the control group $(p=0.004)$. Survival analyses showed that the difference in LC survival between the two groups was entirely attributable to the different proportion of subjects treated with surgery (or to the different proportion of early stage cancers).

The LC incidence pattern in the ITALUNG trial confirmed the high sensitivity of LDCT screening. Indeed, during the screening phase we observed a 55\% excess incidence in the active group followed by a $45 \%$ compensatory reduction in the following years. At the end of follow-up, at 8.5 years, we observed a small statistically non-significant overall deficit of LC cases in the active group (-7\%). The cumulative incidence curves of the NLST showed that catch-up had not been reached at 6.5 years of average follow-up, at which time 64.5 per 10000 LC cases were diagnosed in the LDCT group in comparison with 57.2 in the PA chest radiography group-a $13 \%$ excess $(\mathrm{RR}=1.13 ; 95 \% \mathrm{CI}$ 1.03 to 1.23 ).

After exclusion of LC deaths, the all-cause mortality in the active group decreased by $11 \%$. However, an unexpected mortality reduction in cardiovascular diseases emerged which was statistically significant. This hinted at a possible contribution from LDCT screening or from the clinical encounter with the pulmonologist in determining better management of health conditions frequent in subjects at risk of or affected by smoking-related chronic diseases. In addition, smokers participating in the active arm had slightly higher smoking cessation rates than those in the control group $(21 \%$ vs $18 \%, \mathrm{p}=0.09)$. $^{15}$ The specific impact of LDCT on the management of cardiovascular diseases should be assessed in the pooled analysis of LC screening trials.

The analysis of complications from invasive diagnostic procedures in the ITALUNG study did not support the hypothesis of important adverse effects of LC screening. Indeed, the frequency of adverse events was similar between the two groups, although differently allocated over time.

The ITALUNG study is representative of population-based LC care in central Italy. In an evaluation of the quality of cancer care carried out in Tuscany in 2004, the proportion of LC patients who underwent surgical treatment was $21 \% .^{16}$ Similarly, data from northern Italian cancer registries between 2003 and 2005 showed that surgery was carried out in $18 \%$ of cases and only $10 \%$ of LC cases were diagnosed at stage I. ${ }^{17}$

\section{CONCLUSIONS}

The ITALUNG RCT has, since its inception, been an active member of the USA-EU collaboration ${ }^{18}$ for the harmonisation of screening trials, with the ultimate aim of pooling results after the first outcome of each study has been published. In Europe, in addition to the ITALUNG trial, six other randomised LC screening trials are in progress. ${ }^{19}$ The Dutch-Belgian trial (NELSON), ${ }^{10}$ which is the largest European LC screening trial, has not yet published outcome data. DANTE, ${ }^{20}{ }^{21} \mathrm{MILD}^{22}$ and DLST $^{23} 24$ have reported on their mortality data, which show no difference between the screening and control groups although, recently, pooled data from DANTE and MILD showed non-significant $11 \%$ and $17 \%$ reductions in overall and LC mortality, respectively. ${ }^{25}$ However, none of these published outcome studies alone, including the ITALUNG trial, has sufficient statistical power to detect a real benefit and, for specific analyses, as those assessing benefit for risk profile subgroups. A pooled analysis of all European trials including NELSON and the ongoing UKLS ${ }^{26}$ and LUSI ${ }^{27}$ RCTs is thus becoming a crucial step in assessing the expected benefit of LDCT screening in Europe.

Despite the lack of statistical significance, the ITALUNG trial outcomes suggest that LDCT screening could reduce LC and overall mortality. Together with the high false positive rate, overdiagnosis is the major potentially harmful effect of LDCT screening. Although further studies are necessary to confirm our results, the comparison of the number of LC cases diagnosed in the two groups in the ITALUNG study does not suggest overdiagnosis after an adequate follow-up period. This impression is supported by the results of the survival analysis, which show no difference if surgical treatment is taken into account.

The ITALUNG study has confirmed that LDCT screening, in conjunction with improvement of treatment strategies in early stage LC cases and effective national policies for smoking cessation, is an important tool for the reduction of deaths from LC. ${ }^{3}$ However, before implementation of LC screening programmes in Europe, there are still several critical issues that need to be addressed including optimisation of recruitment, especially for the identification of high-risk subjects (in which biomarkers may have their part to play) and definition of more efficient protocols for nodule management and the optimal screening interval. $^{28} 29$ While waiting for the results of the NELSON and other European LC trials, it is anticipated that a combined rather than an individual study analysis will provide the 
information necessary to support possible modification of public health guidelines in Europe.

\section{Author affiliations}

${ }^{1}$ Clinical Epidemiology Unit, ISPO-Cancer Research and Prevention Institute, Florence, Italy

${ }^{2}$ Pneumonology Department, Careggi Hospital, Florence, Italy

${ }^{3}$ Pulmonary Unit, Cardiothoracic and Vascular Department, University Hospital of Pisa, Pisa, Italy

${ }^{4}$ Radiodiagnostic Unit, ISPO—Cancer Research and Prevention Institute, Florence, Italy

${ }^{5}$ 2nd Radiology Unit, University Hospital of Pisa, Pisa, Italy

${ }^{6}$ Prevention Laboratory Unit, ISPO-Cancer Research and Prevention Institute, Florence, Italy

${ }^{7}$ Radiodiagnostic Section, Department of Experimental and Clinical Biomedical Sciences, University of Florence, Florence, Italy

Collaborators Members of the ITALUNG Working Group: Eugenio Paci (retired, PI), Donella Puliti, Marco Zappa, Cristina Ocello, Gianfranco Manneschi, Carmen Visioli, Giovanna Cordopatri, Francesco Giusti, Ida Esposito: Clinical Epidemiology Unit, ISPO-Cancer Research and Prevention Institute, Florence, Italy. Andrea Lopes Pegna, Roberto Bianchi, Cristina Ronchi: Pneumonology Department, Careggi Hospital, Florence Italy. Laura Carrozzi, Ferruccio Aquilini, Stella Cini, Mariella De Santis, Francesco Pistelli, Filomena Baliva, Antonio Chella, Laura Tavanti: Cardiothoracic and Vascular Department, University Hospital of Pisa, Italy. Michela Grazzini, Florio Innocenti, Ilaria Natali: Pneumonology Department, Hospital of Pistoia, Italy. Mario Mascalchi, Maurizio Bartolucci, Elena Crisci, Agostino De Francisci, Massimo Falchini, Silvia Gabbrielli, Giuliana Roselli, Andrea Masi: Radiology Department, Careggi Hospital, University of Florence, Italy. Fabio Falaschi, Luigi Battola, Annalisa De Liperi, Cheti Spinelli: Radiology Department, University Hospital of Pisa, Italy. Letizia Vannucchi, Alessia Petruzzelli, Davide Gadda, Anna Talina Neri, Franco Niccolai: Radiology Department, Hospital of Pistoia, Italy. Luca Vaggelli: Nuclear Medicine Department, Careggi Hospital, Florence, Italy. Alessandra Vella: Nuclear Medicine Department, Le Scotte University Hospital, Siena, Italy. Francesca Maria Carozzi, Cristina Maddau, Simonetta Bisanzi: Regional Prevention Laboratory Unit, Institute for Oncological Study and Prevention, Florence, Italy. Giulia Picozzi: Radiodiagnostic Unit, ISPO-Cancer Research and Prevention Institute, Florence, Italy. Alberto Janni: Thoracic Surgery Department, Careggi Hospital, Florence, Italy. Alfredo Mussi, Marco Lucchi: Thoracic Surgery Unit, Cardiothoracic and Vascular Department, University Hospital of Pisa, Italy. Camilla Comin: Pathology Department, Careggi Hospital, University of Florence, Italy. Gabriella Fontanini, Adele Renza Tognetti: Pathology Department, University Hospital of Pisa, Italy. Laura lacuzio: University of Modena and Reggio Emilia, Italy. Members of the Cause of Death Review Panel: Adele Caldarella, Alessandro Barchielli, Tuscany Cancer Registry, ISPO—Cancer Research and Prevention Institute; Carlo Alberto Goldoni, Epidemiology Unit, Local Health Unit Modena, Italy.

Contributors EP, ALP, LC, GP, FF, FMC, MM conceived the study design and made significant contributions to the implementation of the study. DP, ALP, LC, GP, $F F, F P, F A, C O$ and MM performed data collection and quality control. DP and EP performed statistical analysis. EP, DP, ALP, LC, GP, FF, MZ, FMC and MM interpreted the results. EP, DP and $M Z$ wrote the manuscript. ALP, LC, GP, FF, FP, MZ, FMC and MM critically reviewed the manuscript.

Funding The study was completely funded by the local government of Tuscany (Decree N. 1014 of 02.25.2004) and by a Research Grant (PRIN 2003) to Professor Mario Mascalchi of the Italian Ministry of Education, University and Research.

Competing interests None declared.

Patient consent Obtained.

Ethics approval The study was approved by the Local Ethics Committee of each participating institution (approval number 29-30 of 30 September 2003; number 23 of 27 October 2003; and number 00028543 of 13 May 2004).

Provenance and peer review Not commissioned; externally peer reviewed.

\section{REFERENCES}

1 Associazione Italiana Registri Tumori (AIRTUM). I numeri del cancro in Italia, 2015. http://www.registri-tumori.it

2 AIRTUM Working Group. Italian cancer figures, report 2011: Survival of cancer patients in Italy. Epidemiol Prev 2011;35(Suppl 3):1-200.

3 Carreras G, Gorini G, Gallus $S$, et al. Predicting the future prevalence of cigarette smoking in Italy over the next three decades. Eur J Public Health 2012;22:699-704.
4 http://www.salute.gov.tit/portale/temi/p2_4.jsp?area=prevenzione (accessed 07/06/2016).

5 National Lung Screening Trial Research TeamAberle DR, Adams AM, Berg CD, et al. Reduced lung-cancer mortality with low-dose computed tomographic screening. N Engl J Med 2011; 365: 395-409.

6 Moyer VA, U.S. Preventive Services Task Force. Screening for lung cancer: U.S. Preventive Services Task Force recommendation statement. Ann Intern Med 2014; 160:330-8.

7 Armaroli P, Villain P, Suonio E, et al. European code against cancer, 4th edition: cancer screening. Cancer Epidemiol 2015;39(Suppl 1): S139-52.

8 Lopes Pegna A, Picozzi G, Mascalchi M, et al., ITALUNG Study Research Group. Design, recruitment and baseline results of the ITALUNG trial for lung cancer screening with low-dose CT. Lung Cancer 2009;64:34-40.

9 Lopes Pegna A, Picozzi G, Falaschi F, et al., ITALUNG Study Research Group. Four-year results of low-dose CT screening and nodule management in the ITALUNG trial. J Thorac Oncol 2013;8:866-75.

10 van lersel $\mathrm{CA}$, de Koning $\mathrm{HJ}$, Draisma $\mathrm{G}$, et al. Risk-based selection from the general population in a screening trial: selection criteria, recruitment and power for the Dutch-Belgian randomised lung cancer multi-slice CT screening trial (NELSON). Int J Cancer 2007;120:868-74.

11 Sobin L, Gospodarowicz M, Wittekind C, eds. International Union Against Cancer (UICC). TNM Classification of Malignant Tumours. 7th edn. Hoboken, NJ: Wiley Blackwell, 2009.

12 Fritz A, Percy C, Jack A, et al, eds. International Classification of Diseases for Oncology. 3rd edn. Geneva: World Health Organization, 2000.

13 Mascalchi M, Lopes Pegna A, Carrozzi L, et al. Does UKLS strategy increase the yield of screen-detected lung cancers? A comparison with ITALUNG. Thorax 2016;71:950-1.

14 Field JK, Duffy SW, Baldwin DR, et al. UK Lung Cancer RCT Pilot Screening Trial: baseline findings from the screening arm provide evidence for the potential implementation of lung cancer screening. Thorax 2016;71:161-70.

15 Pistelli F, Aquilini F, Tavanti L, et al. Predictors of smoking cessation within a lung cancer CT screening trial. Eur Respir J 2011;38(Suppl 55):780s.

16 Caldarella A, Amunni G, Angiolini C, et al. Feasibility of evaluating quality cancer care using registry data and electronic health records: a population-based study. Int I Qual Health Care 2012;24:411-18.

17 Mangone L, Minicozzi P, Vicentini M, et al. Key factors influencing lung cancer survival in northern Italy. Cancer Epidemiol 2013;37:226-32.

18 Field JK, Smith RA, Duffy SW, et al. The Liverpool Statement 2005: priorities for the European Union/United States spiral computed tomography collaborative group. J Thorac Oncol 2006;1:497-8.

19 Field JK, van Klaveren R, Pedersen JH, et al., European Randomized Screening Trial Group. European randomized lung cancer screening trials: post NLST. I Surg Oncol 2013:108:280-6.

20 Infante M, Lutman FR, Cavuto S, et al., DANTE Study Group. Lung cancer screening with spiral CT: baseline results of the randomized DANTE trial. Lung Cancer 2008;59:355-63.

21 Infante M, Cavuto S, Lutman FR, et al., DANTE Study Group. Long-term follow-up results of the DANTE Trial, a randomized study of lung cancer screening with spiral computed tomography. Am J Respir Crit Care Med 2015;191:1166-75.

22 Pastorino $\mathrm{U}$, Rossi M, Rosato $\mathrm{V}$, et al. Annual or biennial $\mathrm{CT}$ screening versus observation in heavy smokers: 5-year results of the MILD trial. Eur I Cancer Prev 2012:21:308-15.

23 Pedersen $\mathrm{JH}$, Ashraf $\mathrm{H}$, Dirksen $\mathrm{A}$, et al. The Danish randomized lung cancer $\mathrm{CT}$ screening trial一overall design and results of the prevalence round. I Thorac Oncol 2009;4:608-14

24 Saghir Z, Dirksen A, Ashraf H, et al. CT screening for lung cancer brings forward early disease. The randomised Danish Lung Cancer Screening Trial: status after five annual screening rounds with low-dose CT. Thorax 2012;67:296-301.

25 Infante M, Sestini S, Galeone C, et al. Lung cancer screening with low-dose spiral computed tomography: evidence from a pooled analysis of two Italian randomized trials. Eur J Cancer Prev 2016

26 Field JK, Duffy SW, Baldwin DR, et al. The UK Lung Cancer Screening Trial: a pilot randomised controlled trial of low-dose computed tomography screening for the early detection of lung cancer. Health Technol Assess 2016;20:1-146.

27 Becker N, Motsch E, Gross ML, et al. Randomized study on early detection of lung cancer with MSCT in Germany: results of the first 3 years of follow-up after randomization. J Thorac Oncol 2015:10: 890-6.

28 Field JK, Devaraj A, Duffy SW, et al. CT screening for lung cancer: is the evidence strong enough? Lung Cancer 2016;91:29-35.

29 Yousaf-Khan U, van der Aalst $\mathrm{C}$, de Jong PA, et al. Final screening round of the NELSON lung cancer screening trial: the effect of a 2.5-year screening interval. Thorax 2017:72:48-56. 